\title{
Preoperative Low Muscle Mass and Low Muscle Quality Negatively Impact on Pulmonary Function in Patients Undergoing Hepatectomy for Hepatocellular Carcinoma
}

\author{
Hisaya Shirai Toshimi Kaido Yuhei Hamaguchi Atsushi Kobayashi \\ Shinya Okumura Siyuan Yao Shintaro Yagi Naoko Kamo Kojiro Taura \\ Hideaki Okajima Shinji Uemoto
}

Division of Hepato-Biliary-Pancreatic and Transplant Surgery, Department of Surgery, Graduate School of Medicine, Kyoto University, Kyoto, Japan

\section{Keywords}

Sarcopenia - Cardiac function · Pulmonary function · Hepatocellular carcinoma · Psoas muscle index $\cdot$ Intramuscular adipose tissue content

\begin{abstract}
Background: Sarcopenia is a prognostic factor for mortality in digestive surgery. However, the correlation between preoperative cardiopulmonary function and sarcopenia in patients undergoing hepatectomy for hepatocellular carcinoma (HCC) remains unclear. Methods: The present study investigated the impact of preoperative sarcopenia on cardiopulmonary function in 402 patients who underwent first hepatectomy for HCC between April 2005 and April 2015. The quantity and quality of skeletal muscle were evaluated using psoas muscle index (PMI) and intramuscular adipose tissue content (IMAC), respectively, as determined from preoperative computed tomography imaging. Correlations between preoperative cardiopulmonary function and sarcopenic factors (PMI and IMAC) were evaluated. Results: No significant correlations were found between left ventricular ejection fraction and the two sarcopenic factors. On the other hand, preoperative vital capacity (VC) and forced expiratory volume in $1 \mathrm{~s}$ $\left(\mathrm{FEV}_{1}\right)$ correlated significantly with PMI $(p<0.001$ each) in males and with IMAC $(p<0.001$ each) in females. Moreover, VC and $\mathrm{FEV}_{1}$ in the preoperative low PMI ( $p<0.001$ each) and high IMAC ( $p=0.002$ and $p<0.001$, respectively) groups were significantly lower than in the normal group in males. In females, VC and $\mathrm{FEV}_{1}$ were significantly lower in the preoperative high IMAC group than in the normal group ( $p<0.001$ each). Conclusion: Preoperative low muscle mass in males and low muscle quality in males and females were significantly associated with pulmonary dysfunction.




\section{Liver Cancer}

\section{Introduction}

Sarcopenia was initially described as an age-related decrease in muscle mass [1] and is currently considered as a syndrome characterized by progressive and generalized loss of skeletal muscle mass and strength due to various causes. The definition of sarcopenia is now proposed to comprise low muscle mass and impaired muscular function [2, 3]. Sarcopenia is thus not only a condition affecting the elderly and can be observed at any age as a result of inflammatory conditions (including malignancies), malnutrition, and disuse. We have so far reported the negative impacts of preoperative sarcopenia on outcomes after living donor liver transplantation [4, 5], hepatocellular carcinoma (HCC) [6, 7], extrahepatic biliary malignancies [8], and pancreatic cancer [9]. Various lines of evidence have also shown that sarcopenia is predictive of mortality in various diseases and after various surgeries [10-13]. Among them, HCC is the sixth most common human cancer and the third most common cause of cancerrelated death [14]. Hepatectomy plays an important role in the treatment of HCC [15]. However, HCC patients with insufficient cardiopulmonary function cannot undergo hepatectomy.

A previous study demonstrated that among patients with chronic heart failure (CHF), patients with muscle wasting showed significantly lower left ventricular ejection fraction (EF) than patients without muscle wasting [16]. Moreover, Moon et al. [17] revealed an association between low muscle mass and pulmonary function in healthy Korean men and women $\geq 65$ years old.

We previously reported the impact of sarcopenia and pulmonary dysfunction in living donor liver transplantation and the impact of pulmonary dysfunction in patients undergoing living donor liver transplantation [18]. Ezaki et al. [19] classified 139 Japanese patients with HCC undergoing hepatectomy into three groups according to age, and revealed that there was a statistically marked decline in the preoperative cardiac and pulmonary function in the group aged $>66$ years. Although they reported that there was no significant difference among the three groups in postoperative complications, mortality, and survival, they did not directly discuss the impact of pulmonary dysfunction in HCC patients. There has been no report directly discussing the impact of pulmonary dysfunction in HCC patients. Moreover, the impact of sarcopenia on cardiopulmonary function in patients undergoing digestive surgery, particularly patients with HCC undergoing hepatectomy, remains unclear. The present study investigated the correlations between preoperative cardiopulmonary function and sarcopenic parameters such as muscle mass and muscle quality in patients undergoing hepatectomy for HCC.

\section{Patients and Methods}

Patients and Data Collection

Between April 2005 and April 2015, a total of 572 patients underwent hepatectomy for HCC at Kyoto University Hospital. Seventy-two patients who did not undergo preoperative plain computed tomography (CT) at the level of the third lumbar vertebra were excluded from this study. Another 12 patients who underwent neither preoperative echocardiography nor preoperative spirometry in our hospital and 86 patients who underwent hepatectomy more than twice were excluded as well. As a result, 402 patients (325 men, 77 women) with results of echocardiography or preoperative spirometry data were registered in this study.

Data of preoperative blood examinations, including complete blood count, serum biochemistry, tumor marker, and indocyanine green retention test at $15 \mathrm{~min}$, were collected. A pathological examination was performed for evaluating tumor size, tumor number, vascular invasion, tumor differentiation, and background liver in accordance with the rules of the Liver Cancer Study Group of Japan.

Echocardiography was performed in 82 patients (63 men, 19 women) and spirometry in 399 patients (323 men, 76 women). Seventy-nine patients ( 61 men, 18 women) underwent both echocardiography and spirometry. Echocardiography alone was performed in 3 patients ( 2 men, 1 woman) and spirometry alone 


\section{Liver Cancer}

in 320 patients (262 men, 58 women). Cardiac functions such as left ventricular EF were measured from preoperative echocardiography. Pulmonary functions such as vital capacity (VC), percentage of predicted VC $(\% \mathrm{VC})$, forced expiratory volume in $1 \mathrm{~s}\left(\mathrm{FEV}_{1}\right)$, and percentage of predicted $\mathrm{FEV}_{1}\left(\% \mathrm{FEV}_{1}\right)$ were determined from spirometry. We defined restrictive ventilatory impairment as $\% \mathrm{VC}<80 \%$ and obstructive ventilatory impairment as $\% \mathrm{FEV}_{1}<70 \%$ of forced VC (FVC), with combined ventilatory impairment then defined as the presence of both restrictive and obstructive ventilatory impairment. All preoperative CT imaging was performed using a multidetector CT scanner (Aquilion 64; Toshiba Medical Systems, Otawara, Japan).

Image Analysis

The quantity of skeletal muscle was indicated by the psoas muscle index (PMI). The quality of skeletal muscle was indicated by the intramuscular adipose tissue content (IMAC). PMI and IMAC were measured from preoperative CT images at the level of the third lumbar vertebra for all patients (Fig. 1). Cross-sectional areas of bilateral psoas muscles were measured by manual tracing (Fig. 1a), and PMI was calculated by normalizing this cross-sectional area of bilateral psoas muscles to the square of the patient's height in meters. IMAC was calculated as previously described by Kitajima et al. [20]: IMAC = mean CT attenuation value (in Hounsfield units) for region of interest in multifidus muscle / mean CT attenuation value for region of interest in subcutaneous fat.

Subfascial muscular tissue in the multifidus muscle on preoperative cross-sectional plain CT at the level of the third lumbar vertebra was precisely traced, and CT attenuation values were measured using the AquariusNET server (TeraRecon, San Mateo, CA, USA) (Fig. 1b). CT attenuation values of subcutaneous fat were measured for four circular regions of interest in subcutaneous fat away from major vessels (Fig. 1c).

\section{Analyzed Parameters}

We analyzed correlations among PMI or IMAC and preoperative cardiopulmonary parameters including $\mathrm{EF}, \mathrm{VC}, \% \mathrm{VC}, \mathrm{FEV}_{1}$, and $\% \mathrm{FEV}_{1}$. Correlations of $\mathrm{PMI}$ and IMAC with preoperative pulmonary functions were examined. We then examined the relationship between preoperative pulmonary function and degrees of depletion of both skeletal muscle mass and quality. We previously reported sex-specific cutoffs for low PMI of $6.36 \mathrm{~cm}^{2} / \mathrm{m}^{2}$ for men and $3.92 \mathrm{~cm}^{2} / \mathrm{m}^{2}$ for women (mean -2 standard deviations [SDs]) [21, 22]. Based on these donor cutoffs (i.e., cutoffs for healthy young adults), we divided the 399 patients who had undergone spirometry into two groups: a preoperative low PMI group showing low muscle mass (mean below -2 SDs), $n=133$ (122 men, 11 women), and a normal PMI group (mean equal to or above -2 SDs), $n=266$ (201 men, 65 women).

As sex-specific cutoffs for low IMAC, we again applied donor cutoffs, using high IMAC cutoffs of -0.358 for men and -0.229 for women (mean +2 SDs) [22]. Based on these values, we divided the 399 patients who had undergone spirometry into another two groups: a preoperative high IMAC group showing low muscle quality (mean below +2 SDs), $n=221$ (174 men, 47 women), and a normal IMAC group (mean equal to or above +2 SDs), $n=178$ (149 men, 29 women). Multiple comparisons of survival curves according preoperative pulmonary function and risk factors for poor survival were then performed.

\section{Statistical Analysis}

Data are presented as mean \pm SD for continuous variables unless otherwise indicated. Continuous variables were analyzed nonparametrically using the Mann-Whitney U test. Categorical variables were compared using the $\chi^{2}$ test or the Fisher exact test, as appropriate. Cumulative overall survival rates were calculated using the Kaplan-Meier method and differences between curves were evaluated using the Wilcoxon test. In survival time analysis according to group, pairwise comparison of each level of group variable was performed using the Holm method and the $p$ value from the Wilcoxon test, and adjusted $p$ values were determined. $p$ values $<0.05$ were considered to indicate statistical significance. All statistical data were generated using the JMP Pro version 12 software (SAS Institute, Cary, NC, USA).

\section{Results}

\section{Patient Characteristics}

The clinicopathological characteristics of the 402 patients are shown in Table 1 . Median patient age was 67 years (range, 33-89 years). Child-Pugh classification was A for 364 patients 


\section{Liver Cancer}

Fig. 1. Cross-sectional computed tomography images at the level of the third lumbar vertebra. a Areas of bilateral psoas muscle were measured by manual tracing. b Subfascial muscular tissue in multifidus muscle was precisely traced. c Four small circles were placed on subcutaneous fat away from major vessels. Regions of interest of the multifidus muscle and subcutaneous fat (Hounsfield units) were measured by the AquariusNET server.

\begin{tabular}{l|l}
\hline Liver Cancer 2018;7:76-89 \\
\hline DOI: 10.1159/000484487 & $\begin{array}{l}\text { @ 2017 S. Karger AG, Basel } \\
\text { www.karger.com/lic }\end{array}$ \\
\hline
\end{tabular}

Shirai et al.: Preoperative Low Muscle Mass and Low Muscle Quality Negatively Impact on Pulmonary Function in Patients Undergoing Hepatectomy for HCC
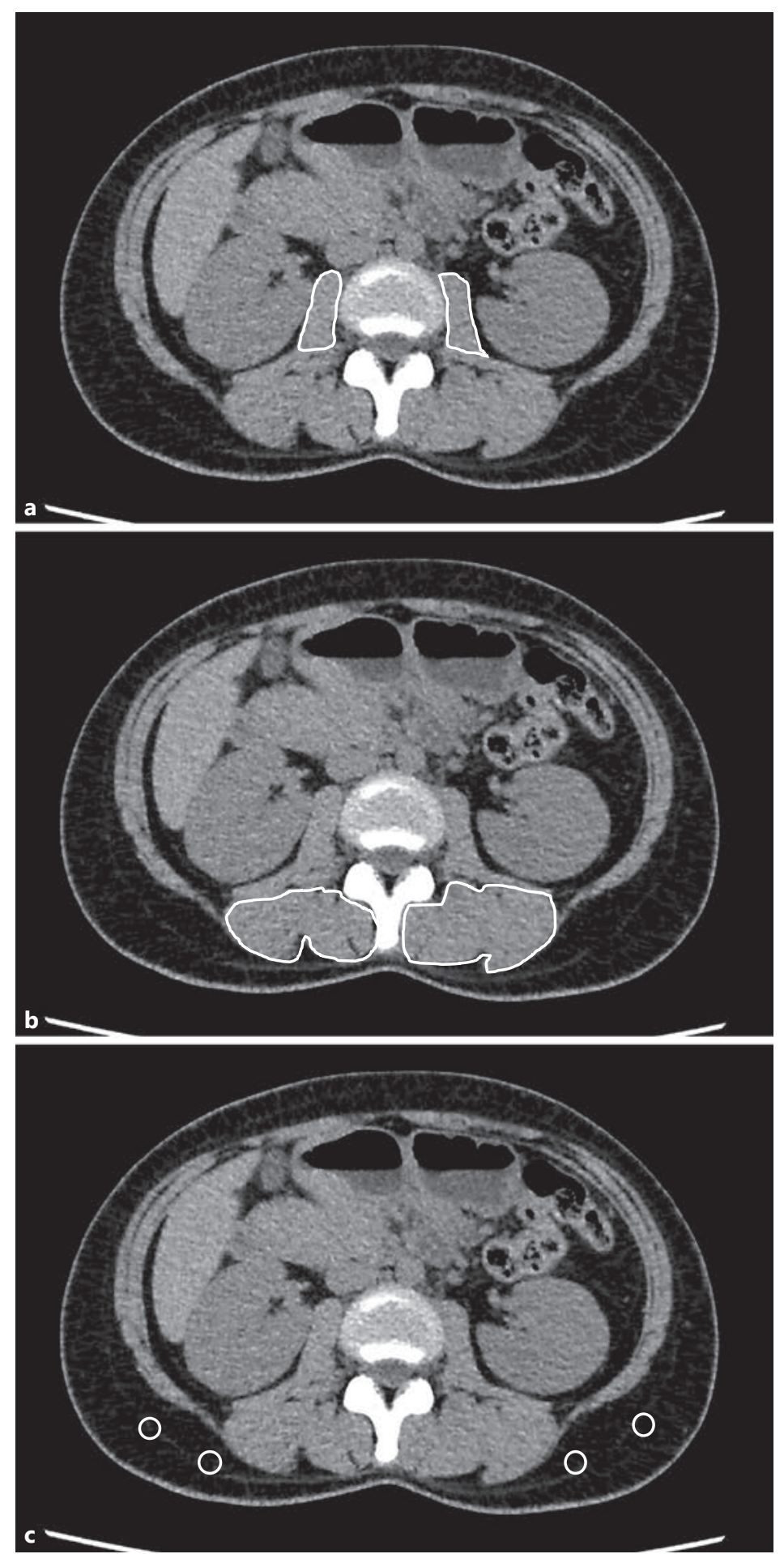

(91\%) and B for 38 patients (9\%). Preoperative median EF was 69.6\% (range, 44.5-84.0\%). Preoperative median VC was $3.32 \mathrm{~L}$ (range, 1.68-5.31 L), and preoperative median \%VC was $104.1 \%$ (range, 65-150.9\%). Preoperative median $\mathrm{FEV}_{1}$ was $2.40 \mathrm{~L} / \mathrm{s}$ (range, 0.86-4.47 L/s), and preoperative median $\% \mathrm{FEV}_{1}$ was $76.3 \%$ (range, $33.3-100.0 \%$ ). 
Shirai et al.: Preoperative Low Muscle Mass and Low Muscle Quality Negatively Impact on Pulmonary Function in Patients Undergoing Hepatectomy for HCC

Table 1. Clinicopathological patient characteristics

\section{Age, years}

Sex (male/female)

Body mass index

Child-Pugh classification $(\mathrm{A} / \mathrm{B} / \mathrm{C})$

Etiology of HCC (HBV and/or HCC/others)

Previous treatment for HCC (yes/no)

Smoking (current/former/never)

Respiratory past history (yes/no)

Pulmonary function (normal/restrictive/obstructive/combined)

Ejection fraction, \%

Indocyanine green retention test at $15 \mathrm{~min}, \%$

Platelet count, $\times 10^{4} / \mathrm{mm}^{3}$

Total bilirubin, $\mathrm{mg} / \mathrm{dL}$

Albumin, g/dL

Alpha-fetoprotein, ng/mL

Des-gamma-carboxy prothrombin, $\mathrm{mU} / \mathrm{L}$

Surgical procedure ( $\geq$ lobectomy/<segmentectomy)

Operative time, min

Operative blood loss, $\mathrm{g}$

TNM stage (I/II/III/IV)

Number of tumors (solitary/multiple)

Differentiation of HCC (good/moderate/poor/unknown)

Microvascular invasion (positive/negative)

Preoperative psoas muscle index

Males

Females

Preoperative intramuscular adipose tissue content

Males

Females
$67.4 \pm 9.5$

$325 / 77$

$23.2 \pm 3.5$

$364 / 38 / 0$

$264 / 138$

$109 / 293$

$118 / 142 / 142$

$63 / 339$

$290 / 21 / 81 / 7$

$69.6 \pm 7.9$

$17.1 \pm 10.1$

$155 \pm 72.7$

$0.9 \pm 0.6$

$3.9 \pm 0.5$

$21.9(0.9-2,873,490)$

$181(8-431,000)$

$138 / 264$

$385 \pm 139.2$

$1,295 \pm 2,312$

$55 / 179 / 116 / 47$

$270 / 132$

$43 / 255 / 90 / 14$

$62 / 340$

$6.908 \pm 1.621$

$5.172 \pm 1.250$

$-0.337 \pm 0.134$

$-0.170 \pm 0.173$

Values are presented as mean \pm standard deviation, median (range), number of patients, or percentage. HBV, hepatitis B virus; HCC, hepatocellular carcinoma; TNM, Tumor Node Metastasis stage (as defined by the Liver Cancer Study Group of Japan).

\section{Correlations between Preoperative EF and Sarcopenic Factors}

For males, EF did not correlate significantly with PMI $(r=-0.190, p=0.135$; online suppl. Fig. 1A; for all online suppl. material, see www.karger.com/doi/10.1159/000484487) or IMAC ( $r=-0.199, p=0.118$; online suppl. Fig. 1B). Likewise, for females, EF did not correlate significantly with PMI ( $r=-0.198, p=0.415$; online suppl. Fig. 1 C) or IMAC ( $r=-0.330, p=$ 0.168 ; online suppl. Fig. 1D).

\section{Correlations between PMI and Preoperative Pulmonary Parameters}

For males, significant but weak positive correlations were observed between PMI and VC $\left(r=0.255, p<0.001\right.$; Fig. 2a) or $\mathrm{FEV}_{1}(r=0.257, p<0.001$; Fig. $2 \mathrm{c})$, but no correlations were evident between PMI and \%VC ( $r=0.149, p=0.007$; Fig. $2 \mathrm{~b})$ or $\% \mathrm{FEV}_{1}(r=0.078, p=0.160$; Fig. 2d). In contrast, for females, PMI was not correlated with any preoperative pulmonary parameters: VC $\left(r=0.038, p=0.743\right.$; Fig. 2e), \%VC $(r=0.006, p=0.962$; Fig. 2f $), \operatorname{FEV}_{1}(r=$ $0.060, p=0.605$; Fig. $2 \mathrm{~g})$, and $\% \mathrm{FEV}_{1}(r=0.052, p=0.656$; Fig. $2 \mathrm{~h})$.

\section{Correlations between IMAC and Preoperative Pulmonary Parameters}

For males, a significant but weak negative correlation was observed between IMAC and $\mathrm{FEV}_{1}(r=0.203, p<0.001$; Fig. $3 \mathrm{c})$, although no other preoperative pulmonary parameters 
Shirai et al.: Preoperative Low Muscle Mass and Low Muscle Quality Negatively Impact on Pulmonary Function in Patients Undergoing Hepatectomy for HCC

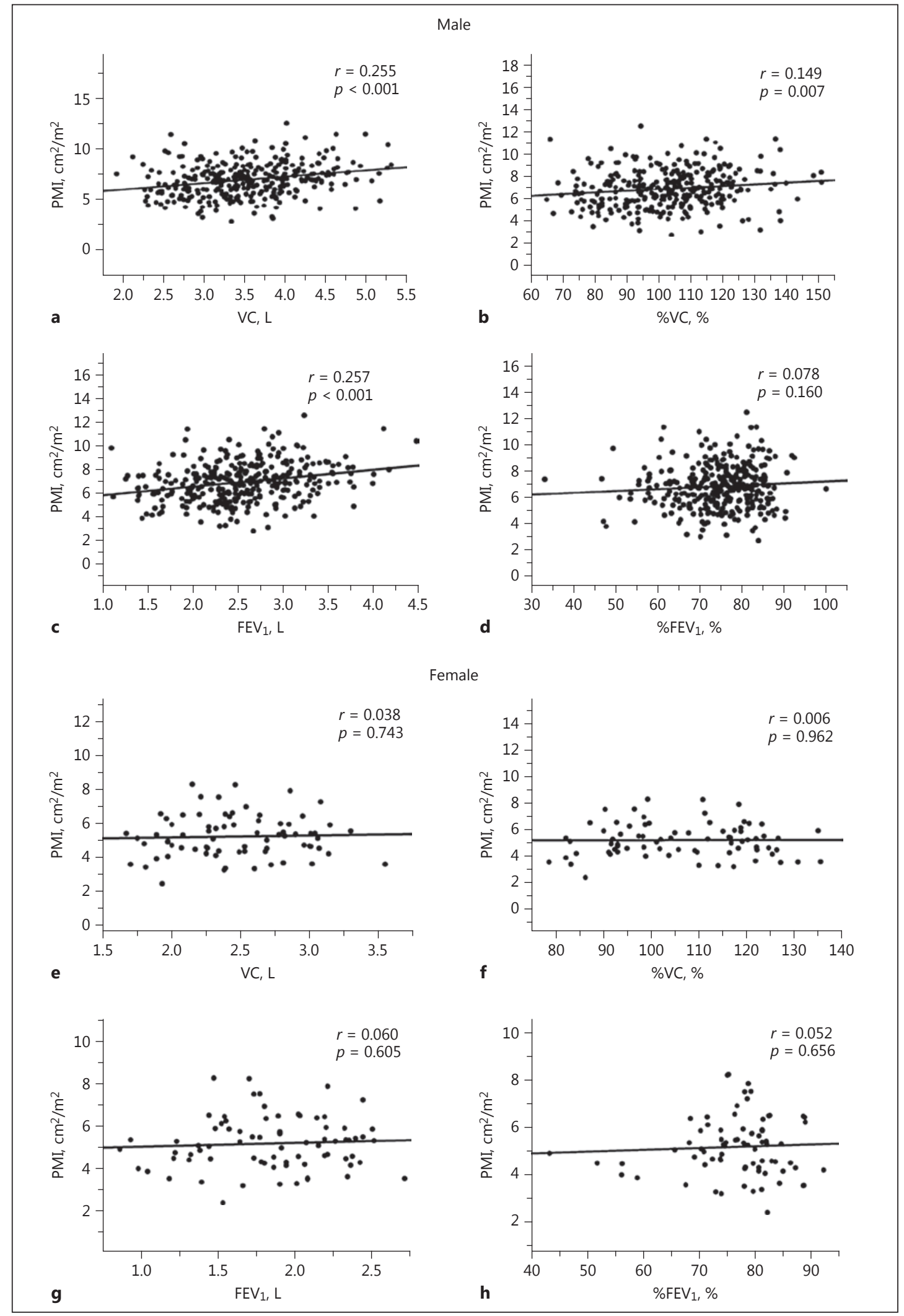

Fig. 2. Correlations between PMI and preoperative pulmonary parameters in males (a: VC; b: \%VC; c: FEV d: $\% \mathrm{FEV}_{1}$ ) and females (e: $\mathrm{VC} ; \mathbf{f}: \% \mathrm{VC} ; \mathbf{g}: \mathrm{FEV}_{1} ; \mathbf{h}: \mathrm{FFEV}_{1}$ ). $\mathrm{FEV}_{1}$, forced expiratory volume in $1 \mathrm{~s} ; \% \mathrm{FEV}_{1}$, percentage of predicted $\mathrm{FEV}_{1}$; PMI, psoas muscle index; VC, vital capacity; \%VC, percentage of predicted VC. 
Shirai et al.: Preoperative Low Muscle Mass and Low Muscle Quality Negatively Impact on Pulmonary Function in Patients Undergoing Hepatectomy for HCC

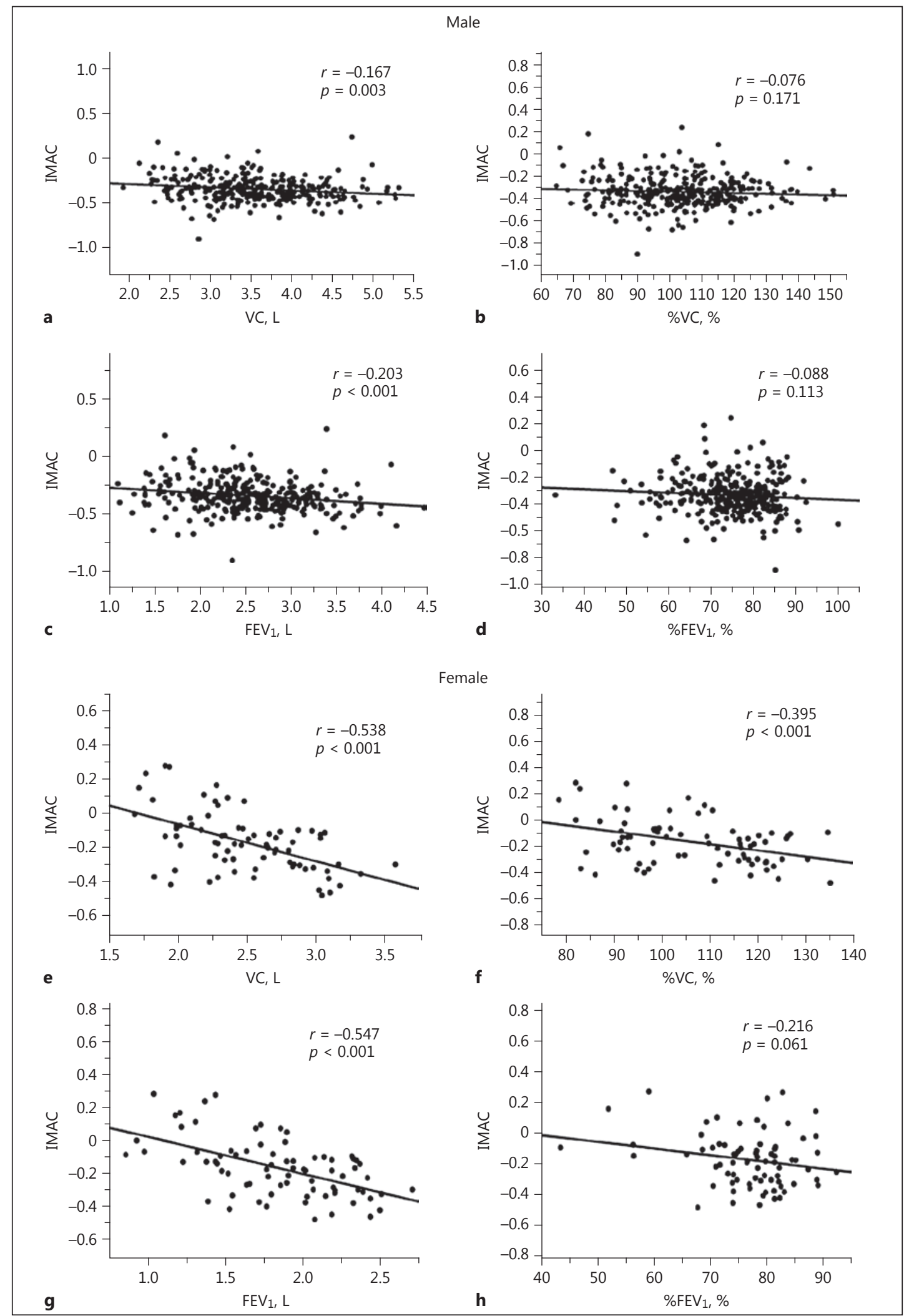

Fig. 3. Correlations between IMAC and preoperative pulmonary parameters in males (a: VC; b: \%VC; $\mathbf{c}: \mathrm{FEV}_{1}$; d: $\% \mathrm{FEV}_{1}$ ) and females (e: $\mathrm{VC} ; \mathbf{f}: \% \mathrm{VC} ; \mathbf{g}: \mathrm{FEV}_{1} ; \mathbf{h}$ : $\% \mathrm{FEV}_{1}$ ). $\mathrm{FEV}_{1}$, forced expiratory volume in $1 \mathrm{~s} ; \%_{\mathrm{FEV}}$, percentage of predicted $\mathrm{FEV}_{1}$; IMAC, intramuscular adipose tissue content; $\mathrm{VC}$, vital capacity; \%VC, percentage of predicted VC. 
Shirai et al.: Preoperative Low Muscle Mass and Low Muscle Quality Negatively Impact on Pulmonary Function in Patients Undergoing Hepatectomy for HCC

Table 2. Baseline characteristics according to low muscle mass and quality in both sexes

\begin{tabular}{|c|c|c|c|c|c|c|}
\hline \multirow{2}{*}{$\begin{array}{l}\text { Pulmonary } \\
\text { parameter }\end{array}$} & \multicolumn{3}{|l|}{ Male $(n=323)$} & \multicolumn{3}{|l|}{ Female $(n=76)$} \\
\hline & $\begin{array}{l}\text { normal muscle } \\
\text { mass }(n=201)\end{array}$ & $\begin{array}{l}\text { Low muscle } \\
\text { mass }(n=122)\end{array}$ & $p$ value & $\begin{array}{l}\text { normal muscle } \\
\text { mass }(n=11)\end{array}$ & $\begin{array}{l}\text { low muscle } \\
\text { mass }(n=65)\end{array}$ & $p$ value \\
\hline VC, L & $3.67 \pm 0.68$ & $3.31 \pm 0.58$ & $<0.001$ & $2.48 \pm 0.40$ & $2.45 \pm 0.58$ & 0.707 \\
\hline$\%$ VC, \% & $105.1 \pm 16.0$ & $99.9 \pm 15.7$ & 0.004 & $106.4 \pm 13.1$ & $107.9 \pm 21.3$ & 0.768 \\
\hline $\mathrm{FEV}_{1}, \mathrm{~L}$ & $2.65 \pm 0.60$ & $2.36 \pm 0.51$ & $<0.001$ & $1.85 \pm 0.42$ & $1.81 \pm 0.51$ & 0.751 \\
\hline$\% \mathrm{FEV}_{1}, \%$ & $75.3 \pm 8.9$ & $74.0 \pm 9.1$ & 0.162 & $76.7 \pm 8.5$ & $78.0 \pm 9.0$ & 0.611 \\
\hline \multirow{2}{*}{$\begin{array}{l}\text { Pulmonary } \\
\text { parameter }\end{array}$} & \multicolumn{3}{|l|}{ Male $(n=323)$} & \multicolumn{3}{|l|}{ Female $(n=76)$} \\
\hline & $\begin{array}{l}\text { normal muscle } \\
\text { quality }(n=149)\end{array}$ & $\begin{array}{l}\text { low muscle } \\
\text { quality }(n=174)\end{array}$ & $p$ value & $\begin{array}{l}\text { normal muscle } \\
\text { quality }(n=29)\end{array}$ & $\begin{array}{l}\text { low muscle } \\
\text { quality }(n=47)\end{array}$ & $p$ value \\
\hline VC, L & $3.65 \pm 0.62$ & $3.42 \pm 0.69$ & 0.002 & $2.69 \pm 0.44$ & $2.34 \pm 0.37$ & $<0.001$ \\
\hline$\% \mathrm{VC}, \%$ & $104.7 \pm 14.6$ & $101.8 \pm 17.1$ & 0.087 & $110.6 \pm 14.5$ & $104.1 \pm 13.9$ & 0.055 \\
\hline $\mathrm{FEV}_{1}, \mathrm{~L}$ & $2.69 \pm 0.60$ & $2.42 \pm 0.55$ & $<0.001$ & $2.07 \pm 0.34$ & $1.70 \pm 0.41$ & $<0.001$ \\
\hline$\% \mathrm{FEV}_{1}, \%$ & $75.7 \pm 8.6$ & $74.0 \pm 9.2$ & 0.102 & $79.6 \pm 5.69$ & $75.3 \pm 9.58$ & 0.073 \\
\hline
\end{tabular}

Values are presented as mean \pm standard deviation. $\mathrm{FEV}_{1}$, forced expiratory volume in $1 \mathrm{~s} ; \% \mathrm{FEV}_{1}$, percentage of predicted $\mathrm{FEV}_{1}$; VC, vital capacity; \%VC, percentage of predicted VC.

correlated significantly with IMAC. For females, IMAC correlated significantly with VC $(r=$ $-0.538, p<0.001$; Fig. 3e), \%VC ( $r=-0.395, p<0.001$; Fig. 3f $)$, and $\operatorname{FEV}_{1}(r=-0.547, p<0.001$; Fig. 3g).

\section{Analysis according to Preoperative PMI Status}

In males, $\mathrm{VC}, \% \mathrm{VC}$, and $\mathrm{FEV}_{1}$ were significantly lower in the preoperative low $\mathrm{PMI}$ group than in the normal PMI group ( $p<0.001, p=0.004$, and $p<0.001$, respectively) (Table 2). In females, preoperative pulmonary functions did not significantly differ between the low PMI and the normal PMI group (Table 2).

\section{Analysis according to Preoperative IMAC Status}

In males, $\mathrm{VC}$ and $\mathrm{FEV}_{1}$ were significantly lower in the preoperative high IMAC group than in the normal IMAC group ( $p=0.002$ and $p<0.001$, respectively) (Table 2). In females, VC and $\mathrm{FEV}_{1}$ were also significantly lower in the high IMAC group than in the normal IMAC group $(p<0.001$ and $p<0.001$, respectively) (Table 2).

Overall Survival Rate after Hepatectomy according to Preoperative Pulmonary Function

The overall survival rate after hepatectomy was significantly lower in patients with preoperative restrictive ventilatory impairment ( $n=21 ; 20$ men, 1 woman) compared with patients with preoperative normal pulmonary function $(n=290 ; 225$ men, 65 women; $p=$ 0.021) (Fig. 4).

\section{Risk Factors for Poor Survival in Patients Undergoing Hepatectomy}

The results of uni- and multivariate analyses of overall survival in patients undergoing hepatectomy for HCC are shown in Table 3. In univariate analysis, previous treatment for HCC, low platelet count $\left(<10 \times 10^{4} / \mathrm{mm}^{3}\right)$, high $\alpha$-fetoprotein $(\geq 20 \mathrm{ng} / \mathrm{dL})$, tumor size $(\geq 5.0 \mathrm{~cm}$ ), number of tumors (solitary), tumor differentiation (poor), TNM stage (stage III or IV), surgical procedure ( $\geq$ lobectomy), operative blood loss ( $\geq 500 \mathrm{~g}$ ), preoperative high IMAC, 
Shirai et al.: Preoperative Low Muscle Mass and Low Muscle Quality Negatively Impact on Pulmonary Function in Patients Undergoing Hepatectomy for HCC

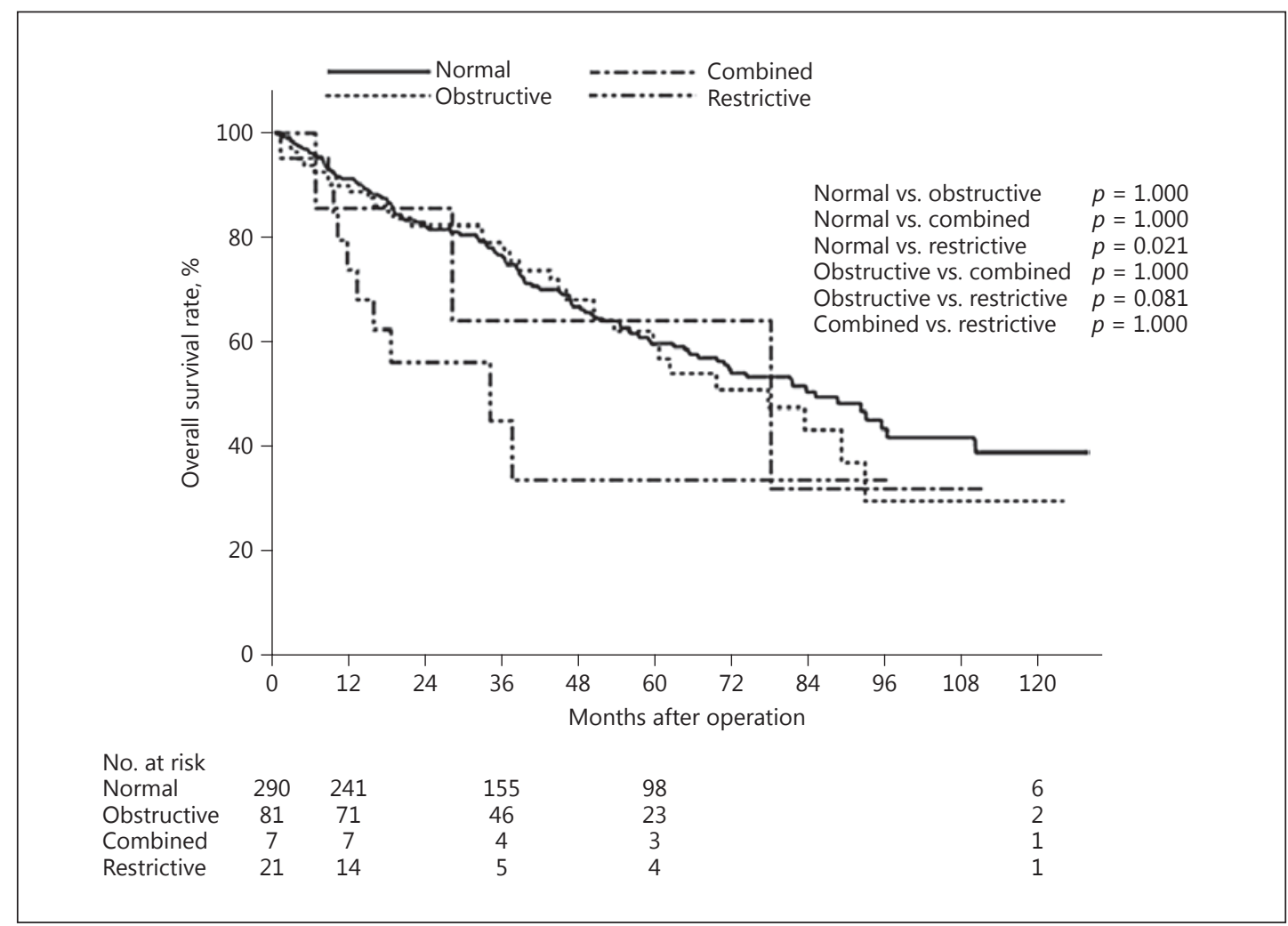

Fig. 4. Overall survival after hepatectomy for patients classified according to pulmonary function.

and restrictive ventilatory impairment were identified as significant poor risk factors among patients undergoing hepatectomy.

Multivariate analysis revealed liver histology (liver fibrosis or cirrhosis), tumor size $(\geq 5.0 \mathrm{~cm}$ ), TNM stage (III or IV), and preoperative high IMAC as independent risk factors, but preoperative restrictive ventilatory impairment was not a significant risk factor even though the overall survival of patients with preoperative restrictive ventilatory impairment was significantly lower than that of patients with normal pulmonary function.

\section{Discussion}

To the best of our knowledge, this retrospective study of 402 patients is the first to investigate the relationship between preoperative cardiopulmonary function and sarcopenic factors in patients undergoing first hepatectomy for HCC. In the present study, low pulmonary function was closely associated with sarcopenic factors such as PMI and IMAC. Pulmonary function progressively decreases after 20-25 years of age [23]. With age, parenchymal lung tissue is lost, chest wall compliance decreases, and intercostal muscle mass and force are reduced as the elastic elements in the lungs degenerate [24]. Moon et al. [17] revealed an association between low muscle mass and pulmonary function in healthy Korean men and women aged $\geq 65$ years. Moreover, Jeon et al. [25] showed that low pulmonary function, as represented by decreased $\mathrm{FEV}_{1}$ or $\mathrm{VC}$, was associated with low muscle mass in older adults. Findings from the present study of patients undergoing hepatectomy for HCC are in line with those reports. 
Shirai et al.: Preoperative Low Muscle Mass and Low Muscle Quality Negatively Impact on Pulmonary Function in Patients Undergoing Hepatectomy for HCC

Table 3. Univariate and multivariate analyses of prognostic factors for survival after hepatectomy

\begin{tabular}{|c|c|c|c|c|c|c|}
\hline \multirow[t]{2}{*}{ Variable } & \multicolumn{3}{|c|}{ Univariate } & \multicolumn{3}{|c|}{ Multivariate } \\
\hline & HR & $95 \% \mathrm{CI}$ & $p$ value & HR & $95 \% \mathrm{CI}$ & $p$ value \\
\hline \multicolumn{7}{|l|}{ Age } \\
\hline$<65$ years $(n=145)$ & 1.000 & (reference) & & & & \\
\hline$\geq 65$ years $(n=257)$ & 1.068 & $0.772-1.489$ & 0.695 & & & \\
\hline \multicolumn{7}{|l|}{ Sex } \\
\hline Male $(n=325)$ & 1.000 & (reference) & & & & \\
\hline Female $(n=77)$ & 1.084 & $0.714-1.592$ & 0.695 & & & \\
\hline \multicolumn{7}{|l|}{ Body mass index } \\
\hline$<25(n=313)$ & 1.000 & (reference) & & & & \\
\hline$\geq 25(n=89)$ & 0.846 & $0.557-1.242$ & 0.402 & & & \\
\hline \multicolumn{7}{|l|}{ Etiology of HCC } \\
\hline HBV and/or HCC $(n=264)$ & 1.000 & (reference) & 0.718 & & & \\
\hline Others $(n=138)$ & 0.842 & $0.592-1.179$ & 0.322 & & & \\
\hline \multicolumn{7}{|l|}{ Previous treatment for $\mathrm{HCC}$} \\
\hline Yes $(n=109)$ & 1.000 & (reference) & & 1.000 & (reference) & \\
\hline No $(n=293)$ & 0.689 & $0.497-0.964$ & 0.030 & 0.715 & $0.494-1.045$ & 0.083 \\
\hline \multicolumn{7}{|l|}{ Platelet count } \\
\hline$<10 \times 10^{4} / \mathrm{mm}^{3}(n=89)$ & 1.000 & (reference) & & 1.000 & (reference) & \\
\hline$\geq 10 \times 10^{4} / \mathrm{mm}^{3}(n=313)$ & 0.543 & $0.389-0.768$ & $<0.001$ & 0.721 & $0.487-1.080$ & 0.111 \\
\hline \multicolumn{7}{|l|}{ ICG R15 } \\
\hline$<15 \%(n=199)$ & 1.000 & (reference) & & & & \\
\hline$\geq 15 \%(n=203)$ & 1.202 & $0.875-1.658$ & 0.256 & & & \\
\hline \multicolumn{7}{|l|}{ Child-Pugh classification } \\
\hline $\mathrm{A}(n=364)$ & 1.000 & (reference) & & 1.000 & (reference) & \\
\hline $\mathrm{B}(n=38)$ & 1.635 & $0.978-2.577$ & 0.060 & 1.484 & $0.855-2.450$ & 0.155 \\
\hline \multicolumn{7}{|l|}{ Alpha-fetoprotein } \\
\hline$<20 \mathrm{ng} / \mathrm{dL}(n=194)$ & 1.000 & (reference) & & 1.000 & (reference) & \\
\hline$\geq 20 \mathrm{ng} / \mathrm{dL}(n=208)$ & 1.865 & $1.345-2.614$ & $<0.001$ & 1.432 & $0.996-2.078$ & 0.052 \\
\hline \multicolumn{7}{|l|}{ Des-gamma-carboxy prothrombin } \\
\hline$<40 \mathrm{mU} / \mathrm{L}(n=104)$ & 1.000 & (reference) & & & & \\
\hline$\geq 40 \mathrm{mU} / \mathrm{L}(n=298)$ & 1.344 & $0.926-2.007$ & 0.123 & & & \\
\hline \multicolumn{7}{|l|}{ Liver histology } \\
\hline Normal liver or chronic hepatitis $(n=196)$ & 1.000 & (reference) & & 1.000 & (reference) & \\
\hline Liver fibrosis or cirrhosis $(n=206)$ & 1.366 & $0.993-1.889$ & 0.055 & 1.518 & $1.059-2.185$ & 0.023 \\
\hline \multicolumn{7}{|l|}{ Tumor size } \\
\hline$<5.0 \mathrm{~cm}(n=250)$ & 1.000 & (reference) & & 1.000 & (reference) & \\
\hline$\geq 5.0 \mathrm{~cm}(n=152)$ & 1.923 & $1.400-2.640$ & $<0.001$ & 1.848 & $1.234-2.775$ & 0.003 \\
\hline \multicolumn{7}{|l|}{ Number of tumors } \\
\hline Solitary $(n=270)$ & 1.000 & (reference) & & 1.000 & (reference) & \\
\hline Multiple $(n=132)$ & 1.773 & $1.283-2.437$ & $<0.001$ & 1.243 & $0.851-1.812$ & 0.260 \\
\hline \multicolumn{7}{|l|}{ Tumor differentiation $(\mathrm{n}=388)$} \\
\hline Good or moderate $(n=298)$ & 1.000 & (reference) & & 1.000 & (reference) & \\
\hline Poor $(n=90)$ & 1.949 & $1.370-2.733$ & $<0.001$ & 1.438 & $0.978-2.086$ & 0.064 \\
\hline \multicolumn{7}{|l|}{ TNM stage } \\
\hline I or II $(n=207)$ & 1.000 & (reference) & & 1.000 & (reference) & \\
\hline III or IV $(n=195)$ & 2.854 & $2.045-4.034$ & $<0.001$ & 2.057 & $1.345-3.155$ & $<0.001$ \\
\hline
\end{tabular}


Shirai et al.: Preoperative Low Muscle Mass and Low Muscle Quality Negatively Impact on Pulmonary Function in Patients Undergoing Hepatectomy for HCC

Table 3 (continued)

\begin{tabular}{|c|c|c|c|c|c|c|}
\hline \multirow[t]{2}{*}{ Variable } & \multicolumn{3}{|c|}{ Univariate } & \multicolumn{3}{|c|}{ Multivariate } \\
\hline & HR & $95 \% \mathrm{CI}$ & $p$ value & HR & $95 \%$ CI & $p$ value \\
\hline \multicolumn{7}{|l|}{ Surgical procedure } \\
\hline$<$ Segmentectomy $(n=264)$ & 1.000 & (reference) & & 1.000 & (reference) & \\
\hline$\geq$ Lobectomy $(n=138)$ & 1.688 & $0.431-0.818$ & 0.002 & 1.152 & $0.766-1.735$ & 0.498 \\
\hline \multicolumn{7}{|l|}{ Operative time } \\
\hline$<360 \min (n=181)$ & 1.000 & (reference) & & 1.000 & (reference) & \\
\hline$\geq 360 \min (n=221)$ & 1.344 & $0.978-1.854$ & 0.069 & 0.904 & $0.625-1.313$ & 0.593 \\
\hline \multicolumn{7}{|l|}{ Operative blood loss } \\
\hline$<500 \mathrm{~g}(n=136)$ & 1.000 & (reference) & & 1.000 & (reference) & \\
\hline$\geq 500 \mathrm{~g}(n=266)$ & 2.041 & $1.041-3.062$ & $<0.001$ & 1.155 & $0.729-1.869$ & 0.546 \\
\hline \multicolumn{7}{|l|}{ Preoperative PMI } \\
\hline Normal $(n=268)$ & 1.000 & (reference) & & & & \\
\hline Low $(n=134)$ & 1.176 & $0.843-1.625$ & 0.337 & & & \\
\hline \multicolumn{7}{|l|}{ Preoperative IMAC } \\
\hline Normal $(n=179)$ & 1.000 & (reference) & & 1.000 & (reference) & \\
\hline $\operatorname{High}(n=223)$ & 1.817 & $1.310-2.545$ & $<0.001$ & 2.030 & $1.421-2.930$ & $<0.001$ \\
\hline \multicolumn{7}{|c|}{ Preoperative pulmonary function $(\mathrm{n}=399)$} \\
\hline Normal $(n=290)$ & 1.000 & (reference) & & 1.000 & (reference) & \\
\hline Restrictive $(n=21)$ & 2.093 & $1.023-3.814$ & 0.044 & 1.569 & $0.733-3.047$ & 0.231 \\
\hline Obstructive $(n=81)$ & 1.110 & $0.740-1.621$ & 0.604 & 1.146 & $0740-1.735$ & 0.532 \\
\hline Combined $(n=7)$ & 1.258 & $0.309-3.339$ & 0.705 & 3.509 & $0.559-12.018$ & 0.152 \\
\hline
\end{tabular}

CI, confidence interval; HBV, hepatitis B virus; HCC, hepatocellular carcinoma; HR, hazard ratio; ICG R15, indocyanine green retention test at $15 \mathrm{~min}$; IMAC, intramuscular adipose tissue content; PMI, psoas muscle index; TNM, Tumor Node Metastasis stage (as defined by the Liver Cancer Study Group of Japan).

In males, VC correlated significantly with PMI (Fig. 2a). Moreover, $\mathrm{FEV}_{1}$ significantly correlated with both PMI (Fig. 2c) and IMAC (Fig. 3c). In contrast, in females, PMI did not correlate with either \%VC or VC, while IMAC correlated significantly with both VC (Fig. 3e) and \%VC (Fig. 3f). FEV 1 only showed a significant correlation with IMAC in females (Fig. 3g). When patients were divided into two groups by PMI using each sex-specific cutoff, most analyses showed significant differences between low PMI and pulmonary functions in males. In contrast, no significant correlations were found in females. As for IMAC, similar significant differences were found in both males and females. We speculate that this difference was related to the lower skeletal muscle mass in females than in males. Moreover, the range of distribution of PMI was narrower in females than in males. This reduction in differences in PMI might have resulted in the lack of significant correlation between PMI and pulmonary function among females. On the other hand, IMAC as a parameter of muscle quality showed no differences in quantity or distribution between males and females. Significant close relationships were thus found between IMAC levels and pulmonary functions in both males and females.

This study showed the discrepancy in the result between $\mathrm{FEV}_{1}$ and $\% \mathrm{FEV}_{1}$ in males (Fig. 2), except for $\% \mathrm{VC}$ in men and the result between $\mathrm{VC}$ and $\% \mathrm{VC}$ as well as $\mathrm{FEV}_{1}$ and $\% \mathrm{FEV}_{1}$ (Fig. 3), except for \%VC in females. \%VC is a derived value of $\mathrm{VC}$, which is usually defined as VC of the patient divided by average VC in the population for any person of similar age, sex, 


\section{Liver

Shirai et al.: Preoperative Low Muscle Mass and Low Muscle Quality Negatively Impact on Pulmonary Function in Patients Undergoing Hepatectomy for HCC

and height. $\% \mathrm{FEV}_{1}$ is defined as $\mathrm{FEV}_{1} / \mathrm{FVC}$ ratio. Therefore, these predicted values or calculated ratios, $\% \mathrm{VC}$ or $\% \mathrm{FEV}_{1}$, might not reflect more directly than absolute values as $\mathrm{VC}$ or $\mathrm{FEV}_{1}$.

In contrast, cardiac function did not correlate with any sarcopenic factors. Lin et al. [26] revealed that age-related cardiac sarcopenia occurs in mice and that left ventricular remodeling due to increased end-diastolic pressure could be one mechanism underlying age-related left ventricular dysfunction. Fülster et al. [16] reported that in patients with CHF, patients with muscle wasting showed significantly lower left ventricular EF than patients without muscle wasting. However, the present study found no significant relationship between EF and sarcopenic factors. This may have been because this study included 175 patients $>70$ years old who could undergo hepatectomy. Patients with heart diseases such as CHF are usually excluded as candidates for hepatectomy because such patients often cannot tolerate an operation under general anesthesia. Such selection biases in the present study might have contributed to the lack of a significant relationship between EF and sarcopenic factors. Moreover, to date no evidence has indicated an association between sarcopenia and cardiac function in healthy elderly individuals. Under extreme conditions such as CHF, sarcopenia might influence cardiac function.

The overall survival rate was significantly lower in patients with preoperative pulmonary restrictive impairment than in those with normal preoperative pulmonary function. Although restrictive impairment was a poor risk factor in univariate but not multivariate analysis, preoperative pulmonary dysfunction could increase the incidence of postoperative morbidities such as atelectasis or pneumonia, leading to worse survival.

Several reports have shown the effectiveness of preoperative rehabilitation in decreasing postoperative complication in patients with esophageal [27], colorectal [28], and lung cancers [29]. Koya et al. [30] demonstrated that therapeutic exercise improved cardiopulmonary function evaluated by a 6-min walking test in HCC patients.

We previously reported the impact of nutritional therapy on prognosis in patients undergoing living donor liver transplantation [4]. Taken together, perioperative nutrition therapy and rehabilitation would be important for patients with preoperative sarcopenia or pulmonary dysfunction undergoing hepatectomy for HCC. We are planning to perform perioperative intervention therapy with respiratory rehabilitation and nutritional therapy using branchedchain amino acids.

Several limitations must be considered for the present study. First, this was a retrospective study conducted in a single institution. Whether pulmonary dysfunction was the cause or result of sarcopenia remains unclear. We speculate that both causal relationships are possible. Preexisting sarcopenia might induce pulmonary dysfunction in some patients, and sarcopenia might develop as a result of pulmonary dysfunction in some patients. We are therefore conducting a prospective analysis to clarify the direction of causality between sarcopenia and lung function by measuring these parameters at various time points before and after hepatectomy. Second, the Asian and European Working Group on Sarcopenia in Older People proposed the inclusion of not only low muscle mass, but also low muscle function in the definition of sarcopenia. However, the present study did not estimate low muscle function (i.e., strength or performance). We are therefore now prospectively investigating the relationship between muscle function and cardiopulmonary functions. Third, selection bias was seen for patient inclusion in the study group. As mentioned above, hepatectomy is not usually performed in patients with CHF or severe pulmonary dysfunction, since such patients are at an unacceptable risk of not tolerating the surgery. Patients with severe cardiopulmonary dysfunction might thus have tended to be excluded from the present study.

In conclusion, the present results show that preoperative low muscle mass and low muscle quality are closely related to pulmonary dysfunction in patients undergoing hepa- 
tectomy. Although analysis of the causal relationships is needed, perioperative pulmonary rehabilitation may be useful to prevent pulmonary complications and improve overall survival after hepatectomy for HCC, especially among patients with sarcopenia.

\section{Statement of Ethics}

All study protocols were approved by the ethics committee of Kyoto University and conducted in accordance with the 1996 revision of the Declaration of Helsinki.

\section{Disclosure Statement}

None of the authors of this paper have any conflicts of interest to declare. No financial support from any source was received for this study.

\section{References}

1 Rosenberg IH: Sarcopenia: origins and clinical relevance. J Nutr 1997;127(5 suppl):990S-991S.

2 Cruz-Jentoft AJ, Baeyens JP, Bauer JM, Boirie Y, Cederholm T, Landi F, Martin FC, Michel JP, Rolland Y, Schneider SM, Topinková E, Vandewoude M, Zamboni M; European Working Group on Sarcopenia in Older People: Sarcopenia: European consensus on definition and diagnosis: report of the European Working Group on Sarcopenia in Older People. Age Ageing 2010;39:412-423.

3 Chen LK, Liu LK, Woo J, Assantachai P, Auyeung TW, Bahyah KS, Chou MY, Chen LY, Hsu PS, Krairit O, Lee JS, Lee WJ, Lee Y, Liang CK, Limpawattana P, Lin CS, Peng LN, Satake S, Suzuki T, Won CW, Wu CH, Wu SN, Zhang T, Zeng P, Akishita M, Arai H: Sarcopenia in Asia: consensus report of the Asian Working Group for Sarcopenia. J Am Med Dir Assoc 2014;15:95-101.

4 Kaido T, Ogawa K, Fujimoto Y, Ogura Y, Hata K, Ito T, Tomiyama K, Yagi S, Mori A, Uemoto S: Impact of sarcopenia on survival in patients undergoing living donor liver transplantation. Am J Transplant 2013;13:1549_ 1556.

5 Hamaguchi Y, Kaido T, Okumura S, Fujimoto Y, Ogawa K, Mori A, Hammad A, Tamai Y, Inagaki N, Uemoto S: Impact of quality as well as quantity of skeletal muscle on outcomes after liver transplantation. Liver Transpl 2014;20:1413-1419.

6 Hamaguchi Y, Kaido T, Okumura S, Ito T, Fujimoto Y, Ogawa K, Mori A, Hammad A, Hatano E, Uemoto S: Preoperative intramuscular adipose tissue content is a novel prognostic predictor after hepatectomy for hepatocellular carcinoma. J Hepatobiliary Pancreat Sci 2015;22:475-485.

7 Kobayashi A, Kaido T, Hamaguchi Y, Okumura S, Taura K, Hatano E, Okajima H, Uemoto S: Impact of postoperative changes in sarcopenic factors on outcomes after hepatectomy for hepatocellular carcinoma. J Hepatobiliary Pancreat Sci 2016;23:57-64.

8 Okumura S, Kaido T, Hamaguchi Y, Fujimoto Y, Kobayashi A, Iida T, Yagi S, Taura K, Hatano E, Uemoto S: Impact of the preoperative quantity and quality of skeletal muscle on outcomes after resection of extrahepatic biliary malignancies. Surgery 2016;159:821-833.

9 Okumura S, Kaido T, Hamaguchi Y, Fujimoto Y, Masui T, Mizumoto M, Hammad A, Mori A, Takaori K, Uemoto S: Impact of preoperative quality as well as quantity of skeletal muscle on survival after resection of pancreatic cancer. Surgery 2015;157:1088-1098.

10 Voron T, Tselikas L, Pietrasz D, Pigneur F, Laurent A, Compagnon P, Salloum C, Luciani A, Azoulay D: Sarcopenia impacts on short- and long-term results of hepatectomy for hepatocellular carcinoma. Ann Surg 2015; 261:1173-1183.

11 Peng PD, van Vledder MG, Tsai S, de Jong MC, Makary M, Ng J, Edil BH, Wolfgang CL, Schulick RD, Choti MA, Kamel I, Pawlik TM: Sarcopenia negatively impacts short-term outcomes in patients undergoing hepatic resection for colorectal liver metastasis. HPB (Oxford) 2011;13:439-446.

12 Otsuji H, Yokoyama Y, Ebata T, Igami T, Sugawara G, Mizuno T, Nagino M: Preoperative sarcopenia negatively impacts postoperative outcomes following major hepatectomy with extrahepatic bile duct resection. World J Surg 2015;39:1494-1500.

13 Miyamoto Y, Baba Y, Sakamoto Y, Ohuchi M, Tokunaga R, Kurashige J, Hiyoshi Y, Iwagami S, Yoshida N, Yoshida M, Watanabe M, Baba H: Sarcopenia is a negative prognostic factor after curative resection of colorectal cancer. Ann Surg Oncol 2015;22:2663-2668.

14 Bray F, Ren JS, Masuyer E, Ferlay J: Global estimates of cancer prevalence for 27 sites in the adult population in 2008. Int J Cancer 2013;132:1133-1145. 
15 Akamatsu N, Cillo U, Cucchetti A, Donadon M, Pinna AD, Torzilli G, Kokudo N: Surgery and hepatocellular carcinoma. Liver Cancer 2016;6:44-50.

16 Fülster S, Tacke M, Sandek A, Ebner N, Tschöpe C, Doehner W, Anker SD, von Haehling S: Muscle wasting in patients with chronic heart failure: results from the Studies Investigating Co-morbidities Aggravating Heart Failure (SICA-HF). Eur Heart J 2013;34:512-519.

17 Moon JH, Kong MH, Kim HJ: Implication of sarcopenia and sarcopenic obesity on lung function in healthy elderly: using Korean National Health and Nutrition Examination Survey. J Korean Med Sci 2015;30:16821688.

18 Shirai H, Kaido T, Hamaguchi Y, Yao S, Kobayashi A, Okumura S, Kamo N, Yagi S, Okajima H, Uemoto S: Preoperative low muscle mass has a strong negative effect on pulmonary function in patients undergoing living donor liver transplantation. Nutrition 2018;45:1-10.

19 Ezaki T, Yukaya H, Ogawa Y: Evaluation of hepatic resection for hepatocellular carcinoma in the elderly. Br J Surg 1987;74:471-473.

20 Kitajima Y, Hyogo H, Sumida Y, Eguchi Y, Ono N, Kuwashiro T, Tanaka K, Takahashi H, Mizuta T, Ozaki I, Eguchi T, Kimura Y, Fujimoto K, Anzai K: Severity of non-alcoholic steatohepatitis is associated with substitution of adipose tissue in skeletal muscle. J Gastroenterol Hepatol 2013;28:1507-1514.

21 Hamaguchi Y, Kaido T, Okumura S, Kobayashi A, Hammad A, Tamai Y, Inagaki N, Uemoto S: Proposal for new diagnostic criteria for low skeletal muscle mass based on computed tomography imaging in Asian adults. Nutrition 2016;32:1200-1205.

22 Hamaguchi Y, Kaido T, Okumura S, Kobayashi A, Shirai H, Yagi S, Kamo N, Okajima H, Uemoto S: Impact of skeletal muscle mass index, intramuscular adipose tissue content, and visceral to subcutaneous adipose tissue area ratio on early mortality of living donor liver transplantation. Transplantation 2017;101:565-574.

23 Sharma G, Goodwin J: Effect of aging on respiratory system physiology and immunology. Clin Interv Aging 2006;1:253-260.

24 Lalley PM: The aging respiratory system - pulmonary structure, function and neural control. Respir Physiol Neurobiol 2013;187:199-210.

25 Jeon YK, Shin MJ, Kim MH, Mok JH, Kim SS, Kim BH, Kim SJ, Kim YK, Chang JH, Shin YB, Kim IJ: Low pulmonary function is related with a high risk of sarcopenia in community-dwelling older adults: the Korea National Health and Nutrition Examination Survey (KNHANES) 2008-2011. Osteoporos Int 2015;26:2423-2429.

26 Lin J, Lopez EF, Jin Y, van Remmen H, Bauch T, Han HC, Lindsey ML: Age-related cardiac muscle sarcopenia: combining experimental and mathematical modeling to identify mechanisms. Exp Gerontol 2008;43:296306.

27 Inoue J, Ono R, Makiura D, Kashiwa-Motoyama M, Miura Y, Usami M, Nakamura T, Imanishi T, Kuroda D: Prevention of postoperative pulmonary complications through intensive preoperative respiratory rehabilitation in patients with esophageal cancer. Dis Esophagus 2013;26:68-74.

28 Mayo NE, Feldman L, Scott S, Zavorsky G, Kim DJ, Charlebois P, Stein B, Carli F: Impact of preoperative change in physical function on postoperative recovery: argument supporting prehabilitation for colorectal surgery. Surgery 2011;150:505-514.

29 Benzo R, Wigle D, Novotny P, Wetzstein M, Nichols F, Shen RK, Cassivi S, Deschamps C: Preoperative pulmonary rehabilitation before lung cancer resection: results from two randomized studies. Lung Cancer 2011;74:441445.

30 Koya S, Kawaguchi T, Hashida R, Goto E, Matsuse H, Saito H, Hirota K, Taira R, Matsushita Y, Imanaga M, Nagamatsu A, Shirono T, Shimose S, Iwamoto H, Niizeki T, Kuromatsu R, Miura H, Shiba N, Torimura T: Effects of in-hospital exercise on liver function, physical ability, and muscle mass during treatment of hepatoma in patients with chronic liver disease. Hepatol Res 2017;47:E22-E34. 